\title{
Discovery of a Vast Ionized Gas Cloud in the M51 System
}

\author{
Aaron E. Watkins ${ }^{1,2}$, J. Christopher Mihos ${ }^{2}$ (D), Matthew Bershady ${ }^{3}$ (D), and Paul Harding ${ }^{2}$ (D) \\ ${ }^{1}$ Astronomy Research Unit, University of Oulu, FIN-90014, Finland \\ ${ }^{2}$ Department of Astronomy, Case Western Reserve University, Cleveland, OH 44106, USA \\ ${ }^{3}$ Department of Astronomy, University of Wisconsin, 475 North Charter Street, Madison, WI 53706, USA \\ Received 2018 March 9; revised 2018 April 4; accepted 2018 April 4; published 2018 May 9
}

\begin{abstract}
We present the discovery of a vast cloud of ionized gas (hereafter, the Cloud) $13^{\prime}$ (32 kpc) north of the interacting system M51. We detected this cloud via deep narrowband imaging with the Burrell Schmidt Telescope, where it appears as an extended, diffuse $\mathrm{H} \alpha$-emitting feature with no embedded compact regions. The Cloud spans $\sim 10^{\prime} \times 3^{\prime}(25 \times 7.5 \mathrm{kpc})$ in size and has no stellar counterpart; comparisons with our previous deep broadband imaging show no detected continuum light to a limit of $\mu_{\lim , B} \sim 30 \mathrm{mag} \operatorname{arcsec}^{-2}$. WIYN ${ }^{4}$ SparsePak observations confirm the Cloud's kinematic association with M51, and the high [N II] $/ \mathrm{H} \alpha$, [S II] $/ \mathrm{H} \alpha$, and $[\mathrm{O}$ I] $/ \mathrm{H} \alpha$ line ratios that we measure imply a hard ionization source such as active galactic nuclei (AGN) photoionization or shock heating rather than photoionization due to young stars. Given the strong [N II] emission, we infer roughly solar metallicity for the Cloud, ruling out an origin due to infall of primordial gas. Instead, we favor models where the gas has been expelled from the inner regions of the M51 system due to tidal stripping or starburst/AGN winds and has been subsequently ionized either by shocks or a fading AGN. This latter scenario raises the intriguing possibility that M51 may be the nearest example of an AGN fossil nebula or light echo, akin to the famous "Hanny's Voorwerp" in the IC 2497 system.
\end{abstract}

Key words: galaxies: individual (M51) - galaxies: interactions - galaxies: jets - intergalactic medium ISM: jets and outflows

\section{Introduction}

The galaxy pair M51 (NGC 5194/5) is perhaps the most iconic interacting system. It has been a subject of study since the $1800 \mathrm{~s}$, its spiral structure serving as a key element in early debates over the true nature of galaxies (Steinicke 2012). It has served, via simulations and observations, as an important dynamical benchmark for studies of tidal tails (e.g., Toomre \& Toomre 1972; Burkhead 1978; Rots et al. 1990; Salo \& Laurikainen 2000) and spiral density waves (e.g., Dobbs et al. 2010). Because of its active star formation (star formation rate $(\mathrm{SFR}) \sim 1.6 M_{\odot} \mathrm{yr}^{-1}$; Kennicutt et al. 2009) and close distance (8.6 Mpc; McQuinn et al. 2016), it is also often used to calibrate star formation tracers and gas density-SFR relations (e.g., Calzetti et al. 2005; Kennicutt et al. 2007). Yet despite this scrutiny, the system continues to yield surprises.

For example, while M51 is known to host extended gaseous tidal debris (Rots et al. 1990), the detailed impact of the interaction and subsequent star formation and nuclear activity on this extended gas is poorly understood. Narrowband imaging by Hoopes et al. (2001) also shows an anomalous "hook" of ionized gas overlapping the companion galaxy, possibly stripped from the primary and shock-heated during the interaction. However, while M51 has been the frequent target of narrowband imaging and spectroscopy (e.g., Hoopes et al. 2001; Thilker et al. 2002; Calzetti et al. 2005; Kennicutt et al. 2007), to date these have lacked the wide field coverage necessary to explore the pair's extreme outskirts where more extended and diffuse ionized gas could reside.

In this Letter, we present the discovery of a new such feature in M51: a large, diffuse circumgalactic cloud of ionized gas

\footnotetext{
4 The WIYN Observatory is a joint facility of the University of WisconsinMadison, Indiana University, the National Optical Astronomy Observatory and the University of Missouri.
}

(hereafter, the Cloud), spanning $\sim 10^{\prime} \times 3^{\prime}(25 \times 7.5 \mathrm{kpc})$ in size and located $\sim 13^{\prime}$ (32 kpc) north of NGC 5194's center. We discovered this Cloud through deep wide-field narrowband imaging using the Burrell Schmidt Telescope at Kitt Peak National Observatory. We present the results of this imaging, as well as follow-up spectroscopy done with the Sparsepak Integral Field Unit at the WIYN $3.5 \mathrm{~m}$ Telescope (Bershady et al. 2004, 2005). We discuss the Cloud's possible origins given its properties and our current understanding of the M51 system.

\section{Observations and Data Reduction}

\subsection{Narrowband Imaging}

We observed M51 with the Burrell Schmidt in the spring of 2015 using two custom $\sim 100 \AA$ wide narrowband filters designed to target $\mathrm{H} \alpha+[\mathrm{N}$ II $]$ emission and the adjacent continuum in nearby $(D<20 \mathrm{Mpc})$ galaxies. We observed M51 only on moonless, photometric nights in March, April, and May. The Schmidt field of view covers $1.65 \times 1.65$; in each filter we imaged M51 in $77 \times 1200 \mathrm{~s}$ images, randomly dithering pointings by $\sim 30^{\prime}$ around the target. We also observed 33 offset night-sky fields alongside $\sim 100$ twilight exposures to build flat fields (described further below; however, for details, see Watkins et al. 2017). Finally, we targeted a variety of spectrophotometric standard stars from Massey et al. (1988) for flux calibration.

We began data reduction with standard bias subtraction and nonlinearity corrections. For flat-fielding, we used a combination of night sky and twilight sky images. Due to the low count rate in the narrowband night-sky images, we combined the twilight sky images to build preliminary flat fields. However, because of the Burrell Schmidt's large field of view, these flats contain strong gradients induced by the twilight sky; we thus 


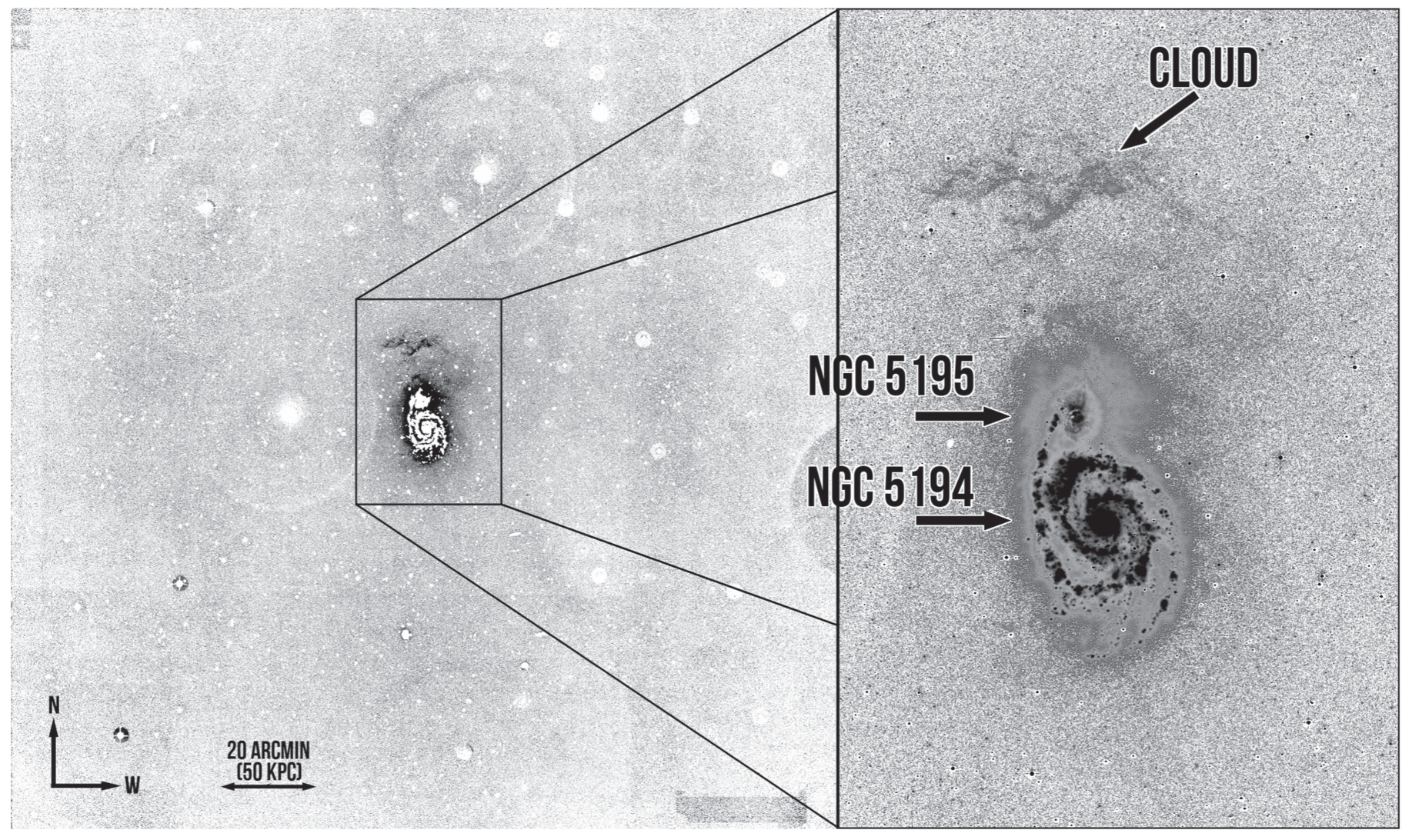

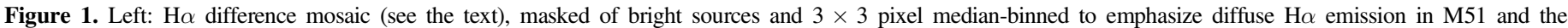

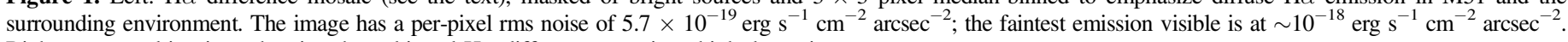
Right: a zoomed-in view, showing the unbinned $\mathrm{H} \alpha$ difference mosaic at high dynamic range.

used the night-sky flats to model and remove these linear gradients (Watkins et al. 2017). To remove mild $(<1 \%$ amplitude) fringing from the on-band exposures, we isolated the fringe pattern from the combined on-band night-sky flats through division by the on-band twilight flat (where the fringe pattern was not visible against ${ }^{5}$ the much brighter flat field), then scaled and subtracted a normalized version of this fringe map from each frame using IRAF's RMFRINGE package.

To reduce the effects of scattered light, we used long (1200 s) exposures of Arcturus to model and remove internal reflections and the extended wings of the point spread function (PSF) from bright $(V \lesssim 10 \mathrm{mag}$ ) stars in each frame (see Slater et al. 2009). For each frame, we then hand-masked all stars, galaxies, and any scattered light artifacts, modeled the remaining sky as a plane, and subtracted it. Finally, we median combined the images to create three final image stacks with total exposure times of $25.6 \mathrm{hr}$ : an on-band mosaic (composed of all on-band exposures), an off-band mosaic (similarly defined), and a "difference" mosaic created by combining individual difference images between on-off pairs observed sequentially in time (Figure 1). We use the difference images to illustrate the morphology of the diffuse $\mathrm{H} \alpha$, but conduct quantitative photometry on the on- and off-band mosaics directly.

\footnotetext{
IRAF is distributed by the National Optical Astronomy Observatory, which is operated by the Association of Universities for Research in Astronomy (AURA), Inc., under cooperative agreement with the National Science Foundation.
}

\subsection{SparsePak Spectroscopy}

We obtained follow-up spectroscopy of the Cloud in 2017 April using SparsePak (Bershady et al. 2004, 2005). SparsePak, comprised of 824 !" 687 diameter fibers, feeds into the WIYN Bench Spectrograph. We centered SparsePak on the region with the brightest emission, where the $72^{\prime \prime} \times 72^{\prime \prime}$ coverage spans the far western end of the Cloud (Figure 2). To target $\mathrm{H} \alpha$ emission, we used the 860 line $\mathrm{mm}^{-1}$ grating in 2 nd order, to cover $6187-7096 \AA$ at a resolution of $\lambda / \delta \lambda=3380\left(\sigma \sim 37 \mathrm{~km} \mathrm{~s}^{-1}\right)$. We fully resolve $\mathrm{H} \alpha$, [N II] 6548/6583 $\AA$, and [S II] 6716/ $6731 \AA$ from each target.

We took $1200 \mathrm{~s}$ exposures of the target, chopping to a nearby sky field on occasion to yield more accurate sky subtraction. Our final object spectrum contains 31 target exposures, with 23 accompanying sky exposures of the same length. We used the IRAF tasks IMCOMBINE and CCDPROC for basic image processing (bias, overscan and dark subtraction, and cosmic ray removal) and DOHYDRA for spectral extraction, channel-tochannel correction (via accompanying dome flat exposures), and wavelength calibration (via accompanying ThAr arc lamp exposures). Calibration data were taken over the course of the run.

Due to the target's low surface brightness, we subtract sky independently of DOHYDRA, augmenting the method described in Appendix D of Bershady et al. (2005) to take advantage of independent sky exposures. Briefly, we first we fit low-order polynomials in wavelength to each extracted fiber aperture in the combined sky frames, rejecting sky-lines via clipping, to produce a sky flat. The flat is normalized and 


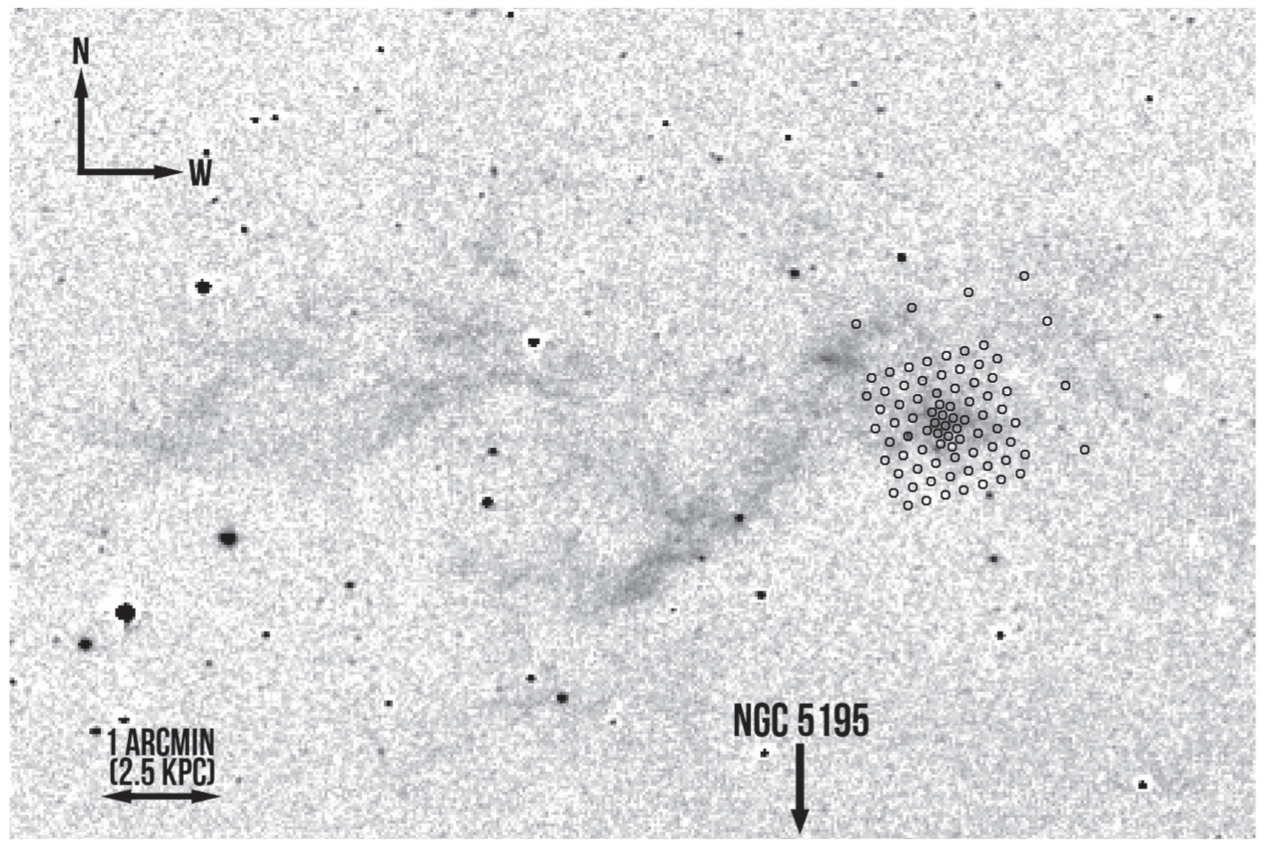

Figure 2. Close-up of the Cloud in the $\mathrm{H} \alpha$ difference image, with the SparsePak fiber array orientation overlaid.

applied to both combined object and sky extracted spectra. The sky spectra are then subtracted from the object spectra, fiber by fiber; this yields excellent subtraction of the sky continuum but leaves residuals in the sky-lines due to different line-to-line temporal variations. To remove these sky-line residuals we fit low-order polynomials, with clipping, in the pseudo-slit dimension for each wavelength channel. The resulting reduced spectrum is shown in Figure 3, coadded from the seven fibers with the strongest [N II] $\lambda 6583 \AA$ signal. The sky spectrum is shown for comparison; note that at this Cloud's velocity, all five targeted lines strongly overlap with telluric line emission.

\section{Results}

We provide the Cloud's basic properties in Table 1, while Figure 1 shows the Cloud's morphology and position relative to M51. It is expansive ( $\sim 25 \mathrm{kpc}$ long), and though it is near M51 on the sky, aside from some very faint $\left(\Sigma_{\mathrm{H} \alpha} \lesssim\right.$ $1.6 \times 10^{-18} \mathrm{erg} \mathrm{s}^{-1} \mathrm{~cm}^{-2} \operatorname{arcsec}^{-2}$ ) diffuse emission northwest of the companion it shows no clear connection to the system. From polygonal aperture photometry (e.g., Watkins et al. 2015) of the narrowband imaging, we measure the Cloud's total $\mathrm{H} \alpha+[\mathrm{N} \mathrm{II}]$ luminosity as $\sim 4 \times 10^{39} \mathrm{erg} \mathrm{s}^{-1}$. From the coadded SparsePak spectra, the Cloud's mean [N II] $(6583+6548) / \mathrm{H} \alpha$ ratio is $\sim 1.48$, hence the Cloud's total $\mathrm{H} \alpha$ luminosity is $\mathrm{L}_{\mathrm{H} \alpha} \sim 1.6 \times 10^{39} \mathrm{erg} \mathrm{s}^{-1}$.

We detect this $\mathrm{H} \alpha$ emission, the [N II] and [S II] doublets, and faint $[\mathrm{OI}] \lambda 6300 \AA$ in seven of the SparsePak fibers; $\mathrm{H} \alpha$ and [N II] $\lambda 6583 \AA$ are visible in an additional 17 fibers. Doing a combined fit to the [N II], [S II], and $\mathrm{H} \alpha$ lines for the seven fibers with the brightest [N II] emission, we measure a heliocentric velocity of $637 \pm 13 \mathrm{~km} \mathrm{~s}^{-1}$; the quoted uncertainty is the standard deviation of the individual fiber measurements, most of which is astrophysical variance in radial velocity. This velocity is well outside the realm of most Milky Way emission, including high-velocity clouds, which typically have velocities $<500 \mathrm{~km} \mathrm{~s}^{-1}$ (and none of which have been discovered so near M51 on the sky; Westmeier 2018). The
Cloud's observed velocity is within the range of $\mathrm{H}$ I velocities observed in the M51 system (Rots et al. 1990), confirming its kinematic association with the interacting pair.

In the left panel of Figure 4, we overlay contours of $\mathrm{H} \alpha$ emission (convolved with a $3 \times 3$ pixel Gaussian kernel to emphasize diffuse features) on a deep Burrell Schmidt $B$-band image of M51 (Watkins et al. 2015). The cloud appears to have no stellar counterpart; it is undetected in the broadband imaging to a limit of $\mu_{B} \sim 30 \mathrm{mag} \operatorname{arcsec}^{-2}$, nor is it detected in our similarly deep Washington $M$ image (Watkins et al. 2015). The three apertures with spectral continuum in Figure 3 each have lineemission consistent with background galaxies. Its non-detection in Washington $M$ also implies a low [O III] $\lambda 5007 \AA$ flux: $<5 \times$ $10^{-17} \mathrm{erg} \mathrm{s}^{-1} \mathrm{~cm}^{-2} \operatorname{arcsec}^{-2}$, or $\log ([\mathrm{O} \mathrm{III]}] / \mathrm{H} \alpha)<-0.64$. The Cloud lies just east of M51's diffuse northwestern stellar plume, and shows morphological contiguity with the northernmost of the bifurcated western streams extending from the companion, the faintest extension of which arcs toward it. We also note a marginal detection of diffuse far-ultraviolet emission near the Cloud (Bigiel et al. 2010), however it is coincident with $12 \mu \mathrm{m}$ emission visible in the WISE Galactic cirrus map (Meisner \& Finkbeiner 2014) of the region, and hence cannot be firmly associated with the Cloud.

The right panel of Figure 4 shows H I contours from the interferometry map of Rots et al. (1990) overlaid on our $\mathrm{H} \alpha$ difference mosaic. While no high column density H I $\left(>3.3 \times 10^{19} \mathrm{~cm}^{-2}\right)$ lies directly coincident with the Cloud, diffuse $\mathrm{H}$ I potentially associated with it is found throughout the region (D. J. Pisano 2018, private communication). We measure a $\sim 40 \mathrm{~km} \mathrm{~s}^{-1}$ gradient across the central fiber bundle, oriented northeast to southwest (with the highest velocities in the east); this orientation is similar to velocity gradients across the two high-velocity (520-660 $\mathrm{km} \mathrm{s}^{-1}$; Rots et al. 1990) H I clouds straddling it, implying a potential connection as well.

The [N II], [S II], and [O I] lines give additional information on the Cloud's ionization source. From the coadded spectrum (Figure 3), we measure $\log ([\mathrm{N}$ II $] \lambda 6584 / \mathrm{H} \alpha)=+0.06, \log$ $([\mathrm{S} \mathrm{II}] \lambda \lambda 6716,31 / \mathrm{H} \alpha)=-0.10$, and $\log ([\mathrm{O} \mathrm{I}] \lambda 6300 / \mathrm{H} \alpha)=$ -0.77 . Variations in the sky subtraction model yield line ratio 

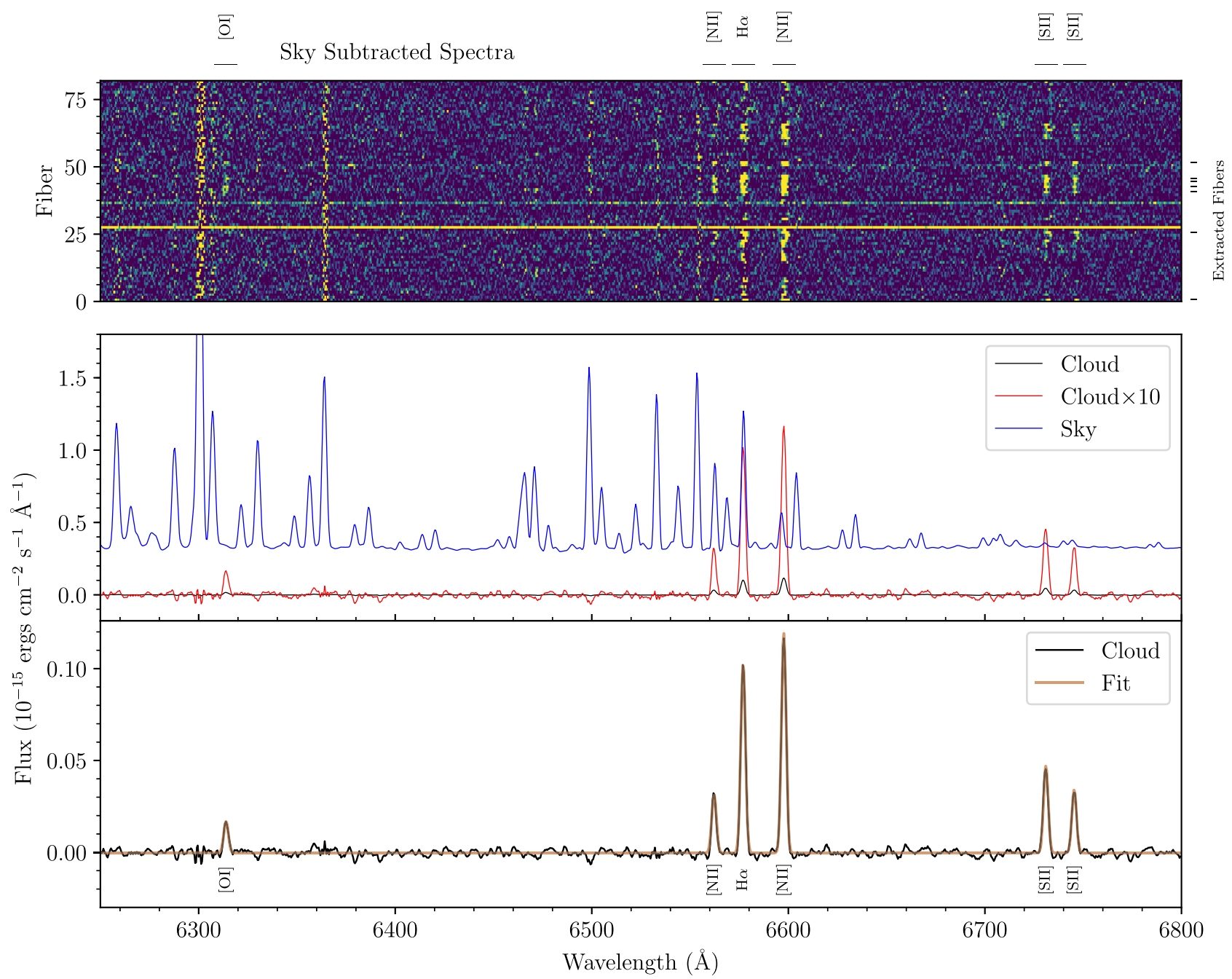

Figure 3. Top: final reduced extracted 2D spectrum of the Cloud. The seven fibers used in co-addition are marked on the right. Middle: final reduced coadded spectrum of the Cloud. The reduced spectrum is shown in black; the red curve scales this spectrum's intensity up by $\times 10$ to show weak emission lines. The foreground sky spectrum (blue) is shown to illustrate the strong overlap between the Cloud's redshifted emission lines and telluric lines. Bottom: fitted spectrum (red) overlaid on the Cloud's reduced spectrum (black).

Table 1

Cloud Properties

\begin{tabular}{ll}
\hline \hline$(\alpha, \delta)_{\text {peak }}$ & $13^{\mathrm{h}} 29^{\mathrm{m}} 51^{\mathrm{s}} 1,+47^{\mathrm{d}} 24^{\mathrm{m}} 34^{\mathrm{s}}$ \\
Dimensions & $10^{\prime} \times 3^{\prime}(25 \times 7.5 \mathrm{kpc})$ \\
$f_{\mathrm{H} \alpha, \text { tot }}$ & $1.8 \times 10^{-13} \mathrm{erg} \mathrm{s}^{-1} \mathrm{~cm}^{-2}$ \\
$L_{\mathrm{H} \alpha, \text { tot }}$ & $1.6 \times 10^{39} \mathrm{erg} \mathrm{s}^{-1}$ \\
Peak $\Sigma_{\mathrm{H} \alpha}$ & $2.2 \times 10^{-16} \mathrm{erg} \mathrm{s}^{-1} \mathrm{~cm}^{-2} \mathrm{arcsec}^{-2}$ \\
$V_{\text {heliocentric }}$ & $637 \pm 13 \mathrm{~km} \mathrm{~s}^{-1}$ \\
$\log ([\mathrm{N} \mathrm{II}] 6748,6583 / \mathrm{H} \alpha)$ & +0.17 \\
$\log ([\mathrm{N} \mathrm{II}] 6583 / \mathrm{H} \alpha)$ & +0.06 \\
$\log ([\mathrm{S} \mathrm{II}] 6717,6731 / \mathrm{H} \alpha)$ & -0.10 \\
$\log ([\mathrm{S} \mathrm{II}] 6717 /[\mathrm{S} \mathrm{II}] 6731)$ & +0.14 \\
$\log ([\mathrm{O} \mathrm{I}] 6300 / \mathrm{H} \alpha)$ & -0.77 \\
$\log ([\mathrm{O} \mathrm{III}] 5007 / \mathrm{H} \alpha)$ & $<-0.64$ \\
\hline
\end{tabular}

uncertainties of $<0.1 \mathrm{dex}$, similar to fiber-to-fiber differences between individual fiber spectra across the field. These line ratios are significantly higher than those in $\mathrm{H}$ II regions, and are more typical of hard photoionization from active galactic nuclei (AGN; e.g., Kewley et al. 2006). In AGN-ionized clouds, the [N II]/H $\alpha$ line ratio is largely a metallicity indicator (e.g., Fu \& Stockton 2009) over a wide range of ionization parameters, and in the case of the M51 Cloud, suggests roughly solar metallicity for the gas.

Alternatively, the gas may be shocked. From MAPPINGS III (Allen et al. 2008) shock models, the Cloud's [N II]/H $\alpha$, [S II]/ $\mathrm{H} \alpha$, and $[\mathrm{OI}] / \mathrm{H} \alpha$ line ratios are well-matched by shock velocities between $\sim 200$ and $300 \mathrm{~km} \mathrm{~s}^{-1}$. In these shock models as well, [N II] $/ \mathrm{H} \alpha$ favors solar metallicity or higher.

In summary, the Cloud's relatively high velocity rules out a chance projection of foreground Milky Way gas, and demonstrates the Cloud's kinematic association with tidally stripped $\mathrm{H}$ I gas in the M51 system. The lack of broadband light and the high $[\mathrm{N} \mathrm{II}] / \mathrm{H} \alpha,[\mathrm{S} \mathrm{II}] / \mathrm{H} \alpha$, and $[\mathrm{O} \mathrm{I}] / \mathrm{H} \alpha$ line ratios argue against ionization from young stars and instead suggest ionization from AGNs or shocks. With the Cloud's association with M51 now well-established, in the next section we explore possible scenarios to explain the Cloud's origin and anomalous line ratios.

\section{Discussion}

While the Cloud's physical association with M51 is well demonstrated, its origin is less clear. M51 is dynamically complex, with active star formation (Kennicutt et al. 2009), 

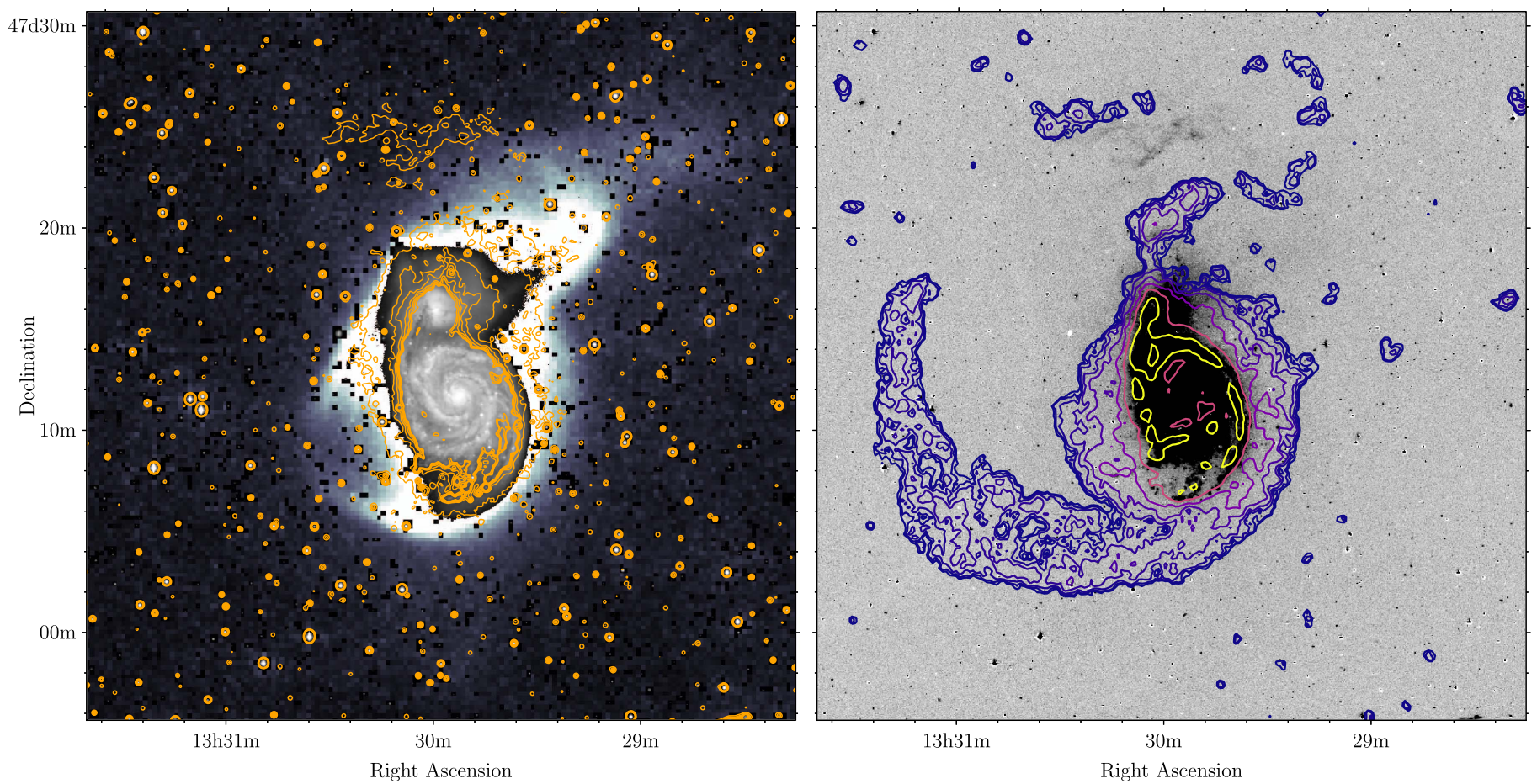

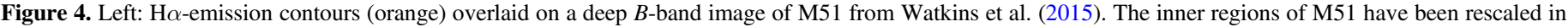

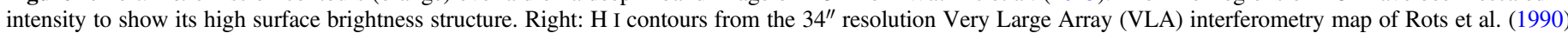
overlaid on our $\mathrm{H} \alpha$ difference mosaic.

numerous and disorganized tidal features (Rots et al. 1990; Watkins et al. 2015), and nuclear activity in both galaxies (Ho et al. 1997; Rampadarath et al. 2018). In such a system, a number of plausible scenarios are available to explain the Cloud's origin, including tidal or ram-pressure stripping during the interaction, infall from the surrounding circumgalactic environment, or ejection from a central starburst or AGN. Constraints on the metallicity and excitation mechanism at work in the Cloud can help differentiate between these various possibilities.

The roughly solar metallicity indicated by the $[\mathrm{N} \mathrm{II}] / \mathrm{H} \alpha$ line ratio constrains the gas' provenance. Such a high metallicity argues against scenarios that involve gas infalling from the circumgalactic environment, which should have lower metallicities ( $~ 1 / 3$ solar; Prochaska et al. 2017). Instead, the Cloud likely comes from M51's similarly high metallicity inner regions (Bresolin et al. 2004). This in turn supports models where the Cloud is tidally stripped gas or, alternatively, gas ejected from M51 via winds from AGN or starburst activity. Strong outflows of gas are seen in many starburst galaxies (e.g., Dahlem et al. 1998) and AGN (e.g., Feruglio et al. 2010), reaching large distances from the host galaxy.

Given M51's ongoing interaction, tidal stripping is perhaps the most obvious origin for the Cloud. This scenario is supported by the Cloud's kinematic contiguity with M51's observed tidal $\mathrm{HI}$, as well as the morphological connection between the Cloud and the NE hook at the end of NGC 5195's western tidal tail. Further information comes from existing numerical simulations that explore M51's interaction history (e.g., Hernquist 1990; Salo \& Laurikainen 2000; Durrell et al. 2003). The Cloud's far northern location and high relative velocity provide the tightest constraints on the models - only the multi-passage model of Salo \& Laurikainen (2000) reproduces any such gas. In this model, this gas is first elevated above NGC 5194's disk plane in response to the companion's initial passage, then is pulled downward along the line of sight by the second encounter. If this model is correct, the Cloud could represent gas initially stripped out and then met by the companion at high velocity during the second passage, resulting in shock-heating (Dopita \& Sutherland 2003). From MAPPINGS III models (Allen et al. 2008), shock velocities between $\sim 200$ and $300 \mathrm{~km} \mathrm{~s}^{-1}$ reproduce the Cloud's observed line ratios, in good agreement with the $174 \pm 13 \mathrm{~km} \mathrm{~s}^{-1}$ line of sight velocity difference between the Cloud and NGC 5194. However, turbulence in shocked gas should also broaden the lines, yet we measure typical line FWHM of only $\sim 60 \mathrm{~km} \mathrm{~s}^{-1}$. This particular model for the M51 system also must necessarily be incomplete as it (and all other simulations to date) fails to reproduce all of the system's extended tidal features (Watkins et al. 2015).

While the Cloud may be tidally stripped, appealing to shock heating as its ionization source may also suffer from a timescale problem. Following Fossati et al. (2016), we can estimate the Cloud's recombination timescale from its density; using an updated derivation of the [S II] density estimator Sanders et al. (2016), the Cloud's $\log ([\mathrm{S}$ II] $\lambda$ 6716/6731) ratio of 0.14 yields $n \sim 50 \mathrm{~cm}^{-3}$, for a recombination time of $\sim 2000$ year, orders of magnitude shorter than the estimated time of last passage (50-500 Myr, e.g., Howard \& Byrd 1990; Salo \& Laurikainen 2000; Durrell et al. 2003). However, these constraints may not be so severe. First, the uncertainty on the [S II] line ratio encompasses the low density limit of the [S II] density estimator, allowing for significantly lower densities and longer recombination times. Second, recently Gavazzi et al. (2017) argued that a diffuse cloud of ionized gas in the Coma Cluster could be as old as 85 Myr. Similarly, a network of shock-excited $\mathrm{H} \alpha$-emitting filaments is seen extending $\sim 120 \mathrm{kpc}$ between the galaxies M86 and NGC 4438 in the 
Virgo Cluster (Kenney et al. 2008); the time since closest approach between these two galaxies is thought to be $\sim 100$ Myr ago. These observations suggest that some mechanism can prolong the lifespans of such clouds well beyond the recombination timescale. In galaxy clusters like Coma or Virgo, this mechanism is likely ongoing ram-pressure effects from the hot intracluster medium. While M51 is not in a cluster environment, numerical simulations suggest that strong galaxy interactions can lead to the formation of hot halo gas (Sinha \& Holley-Bockelmann 2009) and may provide a similar mechanism here.

Alternatively, the high line ratios observed in the Cloud could be due to hard photoionization from either a central AGN or starburst driven winds. "Hanny's Voorwerp," a circumgalactic cloud near the fading AGN IC 2497 (Keel et al. 2012), provides a comparable example of the former; in IC 2497, it is believed that a recent interaction triggered a central AGN that illuminated and ionized the Voorwerp before nuclear activity ceased $\sim 10^{5}$ years ago (evidenced by a radio jet pointing toward the Voorwerp; Keel et al. 2012). A similar scenario may be playing out in M51, where both galaxies host active nuclei (Ho et al. 1997; Rampadarath et al. 2018) and weak nuclear jets (though only NGC 5195's jet is currently aligned with the Cloud; Ford et al. 1985; Rampadarath et al. 2018), although the lack of strong [O III] emission implied by our broadband imaging suggests the Cloud could be older than the Voorwerp (Binette \& Robinson 1987). The currently weak nuclear activity in M51 provides another similarity with the IC 2497 system; following the method employed by Lintott et al. (2009) we find that the current nuclear X-ray luminosity of either galaxy is too faint by four orders of magnitude to account for the Cloud's total $\mathrm{L}_{\mathrm{H} \alpha}$, implying that if the Cloud had been ionized by $\mathrm{AGN}$ emission, the $\mathrm{AGN}$ has since faded significantly. This does not rule out past AGN activity, however, as the strength of such activity, and the jet angle, can evolve on <1 Myr timescales (Denney et al. 2014; Nawaz et al. 2016).

Finally, the Cloud may be the result of starburst driven "superwinds" (Heckman et al. 1990), in which outflows from young massive stars and supernovae in a galaxy's disk generate expanding bubbles of hot gas through the intergalactic medium (IGM). These bubbles can "blow out" and generate localized shocks in density inhomogeneities in the galaxy's gaseous halo tens to hundreds of kiloparsecs from the starburst (Heckman et al. 1990, 2017). One nearby example is the "cap" of ionized gas observed $\sim 10 \mathrm{kpc}$ north of the starburst galaxy M82 (Devine \& Bally 1999; Lehnert et al. 1999), which lies parallel to M82's disk and in direct line with its starburst winds (clearly outlined in $\mathrm{H} \alpha$ and X-ray emission; Lehnert et al. 1999). However, M51 contains no known features indicative of superwinds, its SFR is a factor of two lower than that in M82 (after correcting for extinction; Lehnert et al. 1999; Kennicutt et al. 2009), and the Cloud is much more distant from M51 than the cap is from M82 (>32 kpc, i.e., the projected distance). If the shock front is traveling at $300 \mathrm{~km} \mathrm{~s}^{-1}$ (as we estimate from MAPPINGS III models; Allen et al. 2008), this implies the proposed superwind shock front has been propagating for $>100 \mathrm{Myr}$, significantly longer than starburst durations in most known superwind galaxies (typically of order $10^{7}$ year; Heckman et al. 1990). Therefore, this scenario appears unlikely unless the Cloud's orientation with respect to NGC 5194's disk was significantly more favorable in the past.

\section{Summary}

We report the discovery of a vast, diffuse ionized gas cloud projected $13^{\prime}(32 \mathrm{kpc})$ north of the interacting galaxy pair M51. The Cloud spans $13^{\prime} \times 3^{\prime}(25 \times 7.5 \mathrm{kpc})$ in size, and its systemic velocity $\left(637 \pm 13 \mathrm{~km} \mathrm{~s}^{-1}\right)$ confirms its association with the M51 system. The Cloud has no embedded star formation, and its high $[\mathrm{N} \mathrm{II}] / \mathrm{H} \alpha,[\mathrm{S} \mathrm{II}] / \mathrm{H} \alpha$, and $[\mathrm{O} \mathrm{I}] / \mathrm{H} \alpha$ line ratios suggest AGN photoionization or shock heating. While not directly overlapping with M51's tidal features, the Cloud shows mild evidence of morphological contiguity with the companion galaxy's bifurcated western arm, and kinematic similarity to adjacent tidally stripped $\mathrm{H} \mathrm{I}$.

The strong [N II] emission implies high solar-like metallicities, such that rather than being primordial infall, the Cloud has likely been expelled from the inner disk of NGC 5194 via tidal stripping or AGN/starburst winds. The currently low level of nuclear activity in both galaxies implies that if the Cloud had been ionized by AGN activity, this activity has since faded (a situation similar to IC 2497 and Hanny's Voorwerp; Lintott et al. 2009; Keel et al. 2012). Alternatively, the Cloud may have been ejected by starburst driven "superwinds" (Heckman et al. 1990); however, M51's low SFR relative to known superwind galaxies (e.g., M82; Devine \& Bally 1999; Lehnert et al. 1999), as well as the Cloud's extreme distance from M51 (>32 kpc), suggests that this scenario is less likely. Finally, the gas may be shock heated due to the ongoing interaction between the galaxy pair; if so, it may lend support to the multipassage interaction model of M51 proposed by Salo \& Laurikainen (2000), to date the only such model to produce significant high-velocity gas north of the system.

To discriminate between these various scenarios for the Cloud's origin, additional spectroscopic observations are needed to target emission lines that probe the Cloud's density and temperature structure, better constrain its metallicity, and differentiate between photoionization and shock-heating models for the Cloud. Additional information would come from mapping the line ratios and kinematics of the Cloud across its spatial extent as well.

The Cloud's size and structure-and, perhaps most importantly, the proximity of the M51 system-provide a unique opportunity to study the detailed effects of feedback and ionization on the circumgalactic environments of galaxies. The local universe contains very few known examples of extended diffuse emission sources like the Cloud; each new example provides a wealth of new information about tidal interactions, feedback processes, and the mutual interaction between galaxies and their environment. In particular, if the Cloud is a fossil nebula or echo of strong AGN activity in M51, it would be the most nearby example of a rapidly fading AGN, and also represent a new and critical piece to our understanding of the iconic M51 system.

We thank Heikki Salo and Eija Laurikainen for useful discussions regarding dynamical models of M51, and Tim Heckman, and Bill Keel, and the anonymous referee for useful suggestions. Support for this project was provided by NSF/ AST-1108964 (JCM) and NSF/AST-1517006 (MAB).

Facilities: CWRU : Schmidt, WIYN. 


\section{ORCID iDs}

\section{J. Christopher Mihos 주 https://orcid.org/0000-0002- 7089-8616}

Matthew Bershady (1) https://orcid.org/0000-0002-3131-4374

Paul Harding (1) https://orcid.org/0000-0003-3442-6248

\section{References}

Allen, M. G., Groves, B. A., Dopita, M. A., Sutherland, R. S., \& Kewley, L. J. 2008, ApJS, 178, 20

Bershady, M. A., Andersen, D. R., Harker, J., Ramsey, L. W., \& Verheijen, M. A. W. 2004, PASP, 116, 565

Bershady, M. A., Andersen, D. R., Verheijen, M. A. W., et al. 2005, ApJS, 156,311

Bigiel, F., Leroy, A., Walter, F., et al. 2010, AJ, 140, 1194

Binette, L., \& Robinson, A. 1987, A\&A, 177, 11

Bresolin, F., Garnett, D. R., \& Kennicutt, R. C., Jr. 2004, ApJ, 615, 228

Burkhead, M. S. 1978, ApJS, 38, 147

Calzetti, D., Kennicutt, R. C., Jr., Bianchi, L., et al. 2005, ApJ, 633, 871

Dahlem, M., Weaver, K. A., \& Heckman, T. M. 1998, ApJS, 118, 401

Denney, K. D., De Rosa, G., Croxall, K., et al. 2014, ApJ, 796, 134

Devine, D., \& Bally, J. 1999, ApJ, 510, 197

Dobbs, C. L., Theis, C., Pringle, J. E., \& Bate, M. R. 2010, MNRAS, 403, 625

Dopita, M. A., \& Sutherland, R. S. 2003, Astrophysics of the Diffuse Universe (Berlin: Springer)

Durrell, P. R., Mihos, J. C., Feldmeier, J. J., Jacoby, G. H., \& Ciardullo, R. 2003, ApJ, 582, 170

Feruglio, C., Maiolino, R., Piconcelli, E., et al. 2010, A\&A, 518, L155

Ford, H. C., Crane, P. C., Jacoby, G. H., Lawrie, D. G., \& van der Hulst, J. M 1985, ApJ, 293, 132

Fossati, M., Fumagalli, M., Boselli, A., et al. 2016, MNRAS, 455, 2028

Fraternali, F., Marasco, A., Armillotta, L., \& Marinacci, F. 2015, MNRAS, 447, L70

Fu, H., \& Stockton, A. 2009, ApJ, 690, 953

Gavazzi, G., Consolandi, G., Yagi, M., \& Yoshida, M. 2017, A\&A, 606, A131
Heckman, T. M., Armus, L., \& Miley, G. K. 1990, ApJS, 74, 833

Heckman, T. M., Borthakur, S., Wild, V., Schiminovich, D., \& Bordoloi, R. 2017, ApJ, 846, 151

Hernquist, L. 1990, in Dynamics and Interactions of Galaxies, ed. R. Wielen (Berlin: Springer), 108

Ho, L. C., Filippenko, A. V., \& Sargent, W. L. W. 1997, ApJS, 112, 315

Hoopes, C. G., Walterbos, R. A. M., \& Bothun, G. D. 2001, ApJ, 559, 878

Howard, S., \& Byrd, G. G. 1990, AJ, 99, 1798

Keel, W. C., Lintott, C. J., Schawinski, K., et al. 2012, AJ, 144, 66

Kenney, J. D. P., Tal, T., Crowl, H. H., Feldmeier, J., \& Jacoby, G. H. 2008, ApJL, 687, L69

Kennicutt, R. C., Jr., Calzetti, D., Walter, F., et al. 2007, ApJ, 671, 333

Kennicutt, R. C., Jr., Hao, C.-N., Calzetti, D., et al. 2009, ApJ, 703, 1672

Kewley, L. J., Groves, B., Kauffmann, G., \& Heckman, T. 2006, MNRAS, 372,961

Lehnert, M. D., Heckman, T. M., \& Weaver, K. A. 1999, ApJ, 523, 575

Lintott, C. J., Schawinski, K., Keel, W., et al. 2009, MNRAS, 399, 129

Massey, P., Strobel, K., Barnes, J. V., \& Anderson, E. 1988, ApJ, 328, 315

McQuinn, K. B. W., Skillman, E. D., Dolphin, A. E., Berg, D., \& Kennicutt, R. 2016, ApJ, 826, 21

Meisner, A. M., \& Finkbeiner, D. P. 2014, ApJ, 781, 5

Nawaz, M. A., Bicknell, G. V., Wagner, A. Y., Sutherland, R. S., \& McNamara, B. R. 2016, MNRAS, 458, 802

Prochaska, J. X., Werk, J. K., Worseck, G., et al. 2017, ApJ, 837, 169

Rampadarath, H., Soria, R., Urquhart, R., et al. 2018, MNRAS, 476, 2876

Rots, A. H., Bosma, A., van der Hulst, J. M., Athanassoula, E., \& Crane, P. C. 1990, AJ, 100, 387

Salo, H., \& Laurikainen, E. 2000, MNRAS, 319, 377

Sanders, R. L., Shapley, A. E., Kriek, M., et al. 2016, ApJ, 816, 23

Sinha, M., \& Holley-Bockelmann, K. 2009, MNRAS, 397, 190

Slater, C. T., Harding, P., \& Mihos, J. C. 2009, PASP, 121, 1267

Steinicke, W. 2012, JAHH, 15, 19

Thilker, D. A., Walterbos, R. A. M., Braun, R., \& Hoopes, C. G. 2002, AJ, 124,3118

Toomre, A., \& Toomre, J. 1972, ApJ, 178, 623

Watkins, A. E., Mihos, J. C., \& Harding, P. 2015, ApJL, 800, L3

Watkins, A. E., Mihos, J. C., \& Harding, P. 2017, ApJ, 851, 51

Westmeier, T. 2018, MNRAS, 474, 289 